\title{
PARAMETER IDENTIFICATION IN THE FREQUENCY DOMAIN
}

\author{
H.T. Banks* and Yun Wang* \\ Center for Research in Scientific Computation \\ North Carolina State University \\ Raleigh, NC 27695-8205
}

Revised: March 1993

\begin{abstract}
In this paper, we introduce a method to carry out parameter identification in the frequency domain for distributed parameter systems. Theoretical results related to convergence of approximation ideas for the techniques are presented. An application of the method is illustrated via numerical results for a beam experiment.
\end{abstract}

\section{Introduction}

In studying vibrations of flexible structures, estimation of system parameters using observations in the time domain gave poor results when the observations contained several vibration modes. In response to this difficulty in using time domain optimization techniques, we attempted to carry out identification in the frequency domain. The underlying idea for this procedure involves taking the discrete Fourier transform (DFT) of the data and defining the cost function by using this transformed data and transforms of the model solution. In this paper we outline the theoretical foundations for general frequency domain parameter estimation techniques for second order systems described in terms of sesquilinear forms and operators in a Hilbert space. To illustrate the ideas and techniques, we apply them to the problem of estimating

\footnotetext{
*Research supported in part by the Air Force Office of Scientific Research under grant AFOSR90-0091.
} 
damping parameters in Timoshenko beams.

\section{The Abstract Problem}

Let $V$ and $H$ be complex Hilbert spaces satisfying $V \hookrightarrow H=H^{*} \hookrightarrow V^{*}$ (see [16] for the construction of this so-called Gelfand triple), where we denote their topological duals by $V^{*}$ and $H^{*}$, respectively. Let $Q$ be the admissible parameter metric space with metric $d$. We consider the parameter dependent second order abstract inhomogeneous initial value problem in $V^{*}$

$$
\begin{aligned}
& \ddot{u}(t)+B(q) \dot{u}(t)+A(q) u(t)=f(t) \\
& u(0)=u_{0} \\
& \dot{u}(0)=u_{1}
\end{aligned}
$$

where $A(q)$ and $B(q)$ are parameter dependent differential operators and $q \in Q$. The corresponding variational formulation is given by

$$
\begin{aligned}
& <\ddot{u}(t), \psi>_{V^{*}, V}+\sigma_{1}(u(t), \psi)+\sigma_{2}(\dot{u}(t), \psi)=<f(t), \psi>_{V^{*}, V} \quad \text { for } \psi \in V \\
& u(0)=u_{0} \\
& \dot{u}(0)=u_{1}
\end{aligned}
$$

with $\left\langle\cdot, \cdot>_{V^{*}, V}\right.$ denoting the duality product [16]. We assume that the sesquilinear forms $\sigma_{1}(q)$ and $\sigma_{2}(q)$, where $\sigma_{i}(q): V \times V \rightarrow \mathbb{C}$, satisfy the following conditions:

(A1) Boundedness. There exist $c_{i}>0, i=1,2$ such that for $q \in Q$

$$
\left|\sigma_{i}(q)(\phi, \psi)\right| \leq c_{i}|\phi|_{V} \cdot|\psi|_{V} \quad \text { for } \phi, \psi \in V
$$

(A2) V-Coercivity. There exist $k_{i}>0$ and $\lambda_{i}>0, i=1,2$ such that for $q \in Q$

$$
\operatorname{Re} \sigma_{i}(q)(\phi, \phi) \geq k_{i}|\phi|_{V}^{2}-\lambda_{i}|\phi|_{H}^{2}, \quad \phi \in V
$$

(A3) Continuity. For $q, \tilde{q} \in Q$ and $i=1,2$

$$
\left|\sigma_{i}(q)(\phi, \psi)-\sigma_{i}(\tilde{q})(\phi, \psi)\right| \leq d_{i}(q, \tilde{q})|\phi|_{V}|\psi|_{V}, \phi, \psi \in V
$$

where $d_{i}(q, \tilde{q}) \rightarrow 0$ as $d(q, \tilde{q}) \rightarrow 0$.

If (A1) holds, then $\sigma_{1}, \sigma_{2}$ define operators $A(q), B(q) \in \mathcal{L}\left(V, V^{*}\right)$ by

$$
\begin{aligned}
& \sigma_{1}(q)(\phi, \psi)=<A(q) \phi, \psi>_{V^{*}, V} \\
& \sigma_{2}(q)(\phi, \psi)=<B(q) \phi, \psi>_{V^{*}, V} \quad \text { for } \phi, \psi \in V .
\end{aligned}
$$


In this manner, we have the equivalence of (2.2) and (2.1). The conditions (A1)-(A3) are sufficient to establish well posedness and continuous dependence results for (2.1) and (2.2).

Theorem 1 If the sesquilinear forms $\sigma_{1}$ and $\sigma_{2}$ satisfy conditions (A1)-(A3) with $\sigma_{1}$ symmetric and $f \in L_{2}\left((0, T), V^{*}\right)$, then, for each $w_{0}=\left(u_{0}, u_{1}\right) \in \mathcal{H}=V \times H$, the initial value problem (2.2) has a unique solution $w(t)=(u(t), \dot{u}(t)) \in L_{2}((0, T), V \times$ $V)$. Moreover, this solution depends continuously on $f$ and $w_{0}$ in the sense that the mapping $\left\{w_{0}, f\right\} \rightarrow w=(u, \dot{u})$ is continuous from $\mathcal{H} \times L_{2}\left((0, T), V^{*}\right)$ to $L_{2}((0, T), V \times$ $V)$.

We have in Theorem 1 stated the well posedness of the system (2.2) in a weak variational setting. We can take an alternative (but, as we shall see, equivalent) approach using the theory of semigroups [11], [12]. We can rewrite the second order system (2.1) as a first order system for $w(t)=(u(t), \dot{u}(t))^{T}$ on a product space. We define the product space $\mathcal{V}=V \times V$ in addition to $\mathcal{H}=V \times H$ above and observe that $\mathcal{V}^{*}=V \times V^{*}$ in the Gelfand triple $\mathcal{V} \hookrightarrow \mathcal{H} \hookrightarrow \mathcal{V}^{*}$. The first order system can be written as

$$
\begin{aligned}
& \dot{w}(t)=\mathcal{A} w(t)+F(t) \quad \text { in } \mathcal{V}^{*} \\
& w(0)=w_{0}
\end{aligned}
$$

where $F(t)=(0, f(t))^{T} \in \mathcal{V}^{*}, w_{0}=\left(u_{0}, u_{1}\right)^{T} \in \mathcal{H}$ and

$$
\mathcal{A}=\left(\begin{array}{cc}
0 & I \\
-A & -B
\end{array}\right) \in \mathcal{L}\left(\mathcal{V}, \mathcal{V}^{*}\right)
$$

With the assumptions on $\sigma_{1}$ and $\sigma_{2}$ given in Theorem 1, the operator $\mathcal{A}$ is the infinitesimal generator of an analytic semigroup $\mathcal{T}(t)$ on $\mathcal{V}^{*}$ (see [4] or [2]). Then, by definition, mild solutions of $(2.3)$ in $\mathcal{V}^{*}$ are given by

$$
w(t ; q)=\mathcal{T}(t) w_{0}+\int_{0}^{t} \mathcal{T}(t-s) F(s) d s
$$

Theorem 2 Suppose $w_{0}=\left(u_{0}, u_{1}\right)^{T} \in \mathcal{H}=V \times H, f \in L_{2}\left((0, T), V^{*}\right)$, and sesquilinear forms $\sigma_{1}$ and $\sigma_{2}$ are given as in Theorem 1. Then (2.2) has a unique solution in $L_{2}((0, T), V \times V)$ and it is given by the mild solution $(2.5)$.

For the proofs of both Theorem 1 and Theorem 2 see [4].

For computational efforts in control and estimation of these systems, it is an important result to note that the weak formulation and the semigroup formulation 
yield the same solutions. In actuality, this equivalence can be given under weaker assumptions on $\sigma_{2}$ than (A2). If one relaxes the assumption on $\sigma_{2}$ to $\mathrm{H}$-semiellipticity, one can show that the operator $\mathcal{A}$ of $(2.4)$ defines a $C_{0}$-semigroup on $\mathcal{H}$ which can be extended to a space $\mathcal{Y}$, a proper subset of $\mathcal{V}^{*}$ which contains elements of the form $\left(0, v^{*}\right), v^{*} \in V^{*}$. Then (2.5) can still be used to define mild solutions and the equivalence of solutions from Theorem 2.1 with mild solutions can be established (see [4] for details).

\section{The Optimization Problem}

We formulate the estimation problem as a least squares fit to observations. We seek $\bar{q} \in Q$ which minimizes

$$
J(u, z ; q)=\left|\tilde{C}_{2}\left(\tilde{C}_{1}\left\{u\left(t_{i}, \tilde{x} ; q\right)\right\}-\left\{z\left(t_{i}\right)\right\}\right)\right|^{2}
$$

In $(3.1), u\left(t_{i} ; q\right)$ is the solution to $(2.2)$ (or the first component of the state vector $w(t ; q)$ in $(2.5))$ evaluated at $t_{i}, z\left(t_{i}\right)$ are pointwise time and pointwise space measurements. The operator $\tilde{C}_{1}$ may be in the form of the identity, time differentiation $d / d t$, or time differentiation twice $d^{2} / d t^{2}$, each followed by pointwise evaluation in time and space (at $x=\tilde{x}$ ). The operator $\tilde{C}_{2}$ may be the identity (corresponding to time domain identification procedures) or the Fourier transform (corresponding to identification in the frequency domain). In this paper we only treat the operator $\tilde{C}_{2}$ in form of the Fourier transform. If the measurements are taken with fixed sampling time, i.e., $\Delta t=t_{i}-t_{i-1}=t_{i+1}-t_{i}$ for all $i$ and with a total of $\bar{N}$ samples at the fixed space position $x=\tilde{x}$ (this is often the case in experiments), then the Fourier series coefficients for $0 \leq i \leq \bar{N}$ are given by

$$
\begin{aligned}
& \tilde{C}_{2}\left\{\tilde{C}_{1}\left\{u\left(t_{i}, \tilde{x} ; q\right)\right\}\right\}_{k}=U(k ; q)=\frac{1}{\bar{N}} \sum_{i=0}^{\bar{N}-1} \tilde{C}_{1}\left\{u\left(t_{i}, \tilde{x} ; q\right)\right\} e^{-j k(2 \pi / \bar{N}) i} \\
& \tilde{C}_{2}\left\{z\left(t_{i}\right)\right\}_{k}=Z(k)=\frac{1}{\bar{N}} \sum_{i=0}^{\bar{N}-1} z\left(t_{i}\right) e^{-j k(2 \pi / \bar{N}) i}
\end{aligned}
$$

where $t_{i}=i \cdot \Delta t$ and $k=0,1, \ldots, \bar{N}-1$. In (3.2), we use the generic symbol $U(k ; q)$ to represent the Fourier coefficients of the transform of $\tilde{C}_{1}\{u(\cdot, \tilde{x} ; q)\}$ for all three forms of $\tilde{C}_{1}$. With $\Delta t$ as the sampling time, the $k^{t h}$ value of the frequency corresponding to the $k^{t h}$ coefficient is given by

$$
\mathrm{f}_{k}=\frac{1}{\Delta t \cdot \bar{N}} k
$$


and the corresponding magnitudes are given by $|U(k ; q)|$ and $|Z(k)|$.

We assume that there are a finite and distinct number $(<\bar{N})$ of "spikes" among the $Z(k)$. Since each spike can be described by its frequency (corresponding to its index), magnitude and width of the spike, we will make some modifications to the cost function (3.1) when $\tilde{C}_{2}$ is the Fourier transform. Let $\bar{N}_{M}$ be the number of spikes among the $Z(k)$. We shall assume

(A4) The number of spikes of the solution $U(k ; q)$ is the same as $\bar{N}_{M}$.

After reindexing coefficients of spikes among the $Z(k)$ and $U(k ; q)$ and denoting the indices by $k_{\ell}^{z}, k_{\ell}^{u}$ for $\ell=1, \ldots, \bar{N}_{M}$ with $0 \leq k_{\ell}^{z}, k_{\ell}^{u} \leq \bar{N}-1$, the frequency domain cost function can be more appropriately expressed by

$$
\begin{aligned}
\hat{J}(q) & =\hat{J}(u, z ; q) \\
& =\sum_{\ell=1}^{\bar{N}_{M}}\left(\epsilon_{1}\left|\mathrm{f}_{k_{\ell}^{u}}(q)-\mathrm{f}_{k_{\ell}}\right|^{2}+\epsilon_{2} \sum_{j=-n_{\ell}}^{N_{\ell}}|| U\left(k_{\ell}^{u}+j ; q\right)|-| Z\left(k_{\ell}^{z}+j\right)||^{2}\right),
\end{aligned}
$$

where $\epsilon_{1}, \epsilon_{2}$ are weight constants, and $n_{\ell}, N_{\ell}$ are certain lower and upper limits associated with the width (or the support) of the $\ell^{t h}$ spike. The first part of the cost function (3.5) is related to the frequencies and the second part is related to the magnitude and the width of each spike. The limits $n_{\ell}$ and $N_{\ell}$ depend on the $\ell^{t h}$ spike and are chosen so that $n_{\ell}$ and $N_{\ell}$ are the last $i$ and last $j$ respectively, for which the following conditions are satisfied:

$$
\left|Z\left(k_{\ell}^{k}-i\right)\right| \geq 20 \%\left|Z\left(k_{\ell}^{z}\right)\right|
$$

for $i=1,2, \ldots, n_{\ell}$, and

$$
\left|Z\left(k_{\ell}^{z}+j\right)\right| \geq 20 \%\left|Z\left(k_{\ell}^{z}\right)\right|
$$

for $j=1,2, \ldots, N_{\ell}$. The motivation behind our choice related to the width of the spike is that in traditional modal analysis, the width at approximately $30 \%$ of the peak value of the spike is used to estimate the damping ratio for the $\ell^{\text {th }}$ mode. Hence taking a conservative approach and using the width at $20 \%$ of the peak value should guarantee the inclusion of substantial damping information in the Fourier coefficients.

If the parameter $\bar{q}$ minimizes (3.5), then we shall take $\bar{q}$ as the estimate of the parameter which best describes the system in the frequency domain, i.e. the least squares fit of the model to data in the frequency domain sense. Hereafter, we interpret (3.1) as (3.5) whenever $\tilde{C}_{2}$ is the Fourier transform. 


\section{Approximation Technique}

The minimization in our parameter estimation problem involves an infinite dimensional state space governed by (2.1). For computational purpose, finite dimensional approximations are necessary. To make these approximations, we first select a sequence of finite dimensional spaces $H^{N}$ which are subspaces of $H$. We define orthogonal projections $P_{H}^{N}: H \rightarrow H^{N}, P_{V}^{N}: V \rightarrow H^{N}$, and choose the finite dimensional spaces $\mathcal{H}^{N}=H^{N} \times H^{N}$. We denote the orthogonal projections of $\mathcal{H}=V \times H$ onto $\mathcal{H}^{N}$ by $P_{\mathcal{H}}^{N}$. For convenience, we will hereafter restrict our consideration to the case where $f \in C^{1}([0, T] ; V)$. Then the approximating estimation problems with finite dimensional state spaces can be stated as finding $q \in Q$ which minimizes

$$
J^{N}\left(u^{N}, z ; q\right)=\left|\tilde{C}_{2}\left(\tilde{C}_{1}\left\{u^{N}\left(t_{i}, \tilde{x} ; q\right)\right\}-\left\{z\left(t_{i}\right)\right\}\right)\right|^{2}
$$

or

$$
\begin{aligned}
\hat{J}(q) & =\hat{J}^{N}\left(u^{N}, z ; q\right) \\
& =\sum_{\ell=1}^{\bar{N}_{M}}\left(\epsilon_{1}\left|\mathrm{f}_{k_{\ell}^{u^{N}}}(q)-\mathrm{f}_{k_{\ell}^{z}}\right|^{2}+\epsilon_{2} \sum_{j=-n_{\ell}}^{N_{\ell}}|| U^{N}\left(k_{\ell}^{u^{N}}+j ; q\right)|-| Z\left(k_{\ell}^{z}+j\right)||^{2}\right) .
\end{aligned}
$$

In (4.1) and (4.2), $u^{N}$ is an approximate solution which satisfies a readily-solved finite dimensional system approximating (2.1) given by

$$
\begin{aligned}
& \ddot{u}^{N}(t)+B^{N}(q) \dot{u}^{N}(t)+A^{N}(q) u^{N}(t)=P_{H}^{N} f(t) \\
& u^{N}(0)=P_{V}^{N} u_{0} \\
& \dot{u}^{N}(0)=P_{H}^{N} u_{1},
\end{aligned}
$$

or equivalently the first coordinate of the state vector

$$
w^{N}(t ; q)=\mathcal{T}^{N}(t) P_{\mathcal{H}}^{N} w_{0}+\int_{0}^{t} \mathcal{T}^{N}(t-s) P_{\mathcal{H}}^{N} F(s) d s
$$

Here $A^{N}$ and $B^{N}$ are Galerkin approximations to $A$ and $B$, respectively, and $\mathcal{T}^{N}(t)$ is the obvious corresponding approximation to $\mathcal{T}(t)$, the semigroup of $(2.5)$ generated by the operators of (2.4). Moreover, $U^{N}(k ; q)$, for $k=0,1, \ldots, \bar{N}-1$, in (4.2) is given by

$$
U^{N}(k ; q)=\frac{1}{\bar{N}} \sum_{i=0}^{\bar{N}-1} \tilde{C}_{1}\left\{u^{N}\left(t_{i}, \tilde{x} ; q\right)\right\} e^{-j k(2 \pi / \bar{N}) i}
$$

and $\mathrm{f}_{k_{\ell}^{u^{N}}}$ is defined in the same manner as $f_{k_{\ell}^{u}}$.

In (4.2), we have assumed that the number of "spikes" present in the approximate solution is the same as $\bar{N}_{M}$, the number of "spikes" in the data $z$. If one chooses 
$N$ such that $N \geq 2 \bar{N}_{M}$, then this assumption, which is (A4) for the approximation problems, is guaranteed.

Solving the estimation problems with finite dimensional state spaces, we obtain a sequence of estimates $\left\{\bar{q}^{N}\right\}$. To obtain parameter estimate convergence and continuous dependence (with respect to the observations $\left\{z\left(t_{i}\right)\right\}$ ) results, when $\tilde{C}_{2}$ is the Fourier transform operator, it has been shown in [1], [5] that it suffices, under the assumption that $Q$ is a compact set, to argue: for arbitrary $\left\{q^{N}\right\} \subset Q$ with $q^{N} \rightarrow q$, we have

$$
\tilde{C}_{2} \tilde{C}_{1} u^{N}\left(t ; q^{N}\right) \rightarrow \tilde{C}_{2} \tilde{C}_{1} u(t ; q)
$$

for each $t$.

We first observe that the "solutions" $U^{N}\left(k ; q^{N}\right)$ corresponding to the approximating systems provide approximate solutions to the original system "solutions" $U(k ; q)$.

Theorem 3 Suppose $\left\{q^{N}\right\} \subset Q$ is an arbitrary sequence with $q^{N} \rightarrow \bar{q}$ as $N \rightarrow \infty$. Let $U^{N}\left(k ; q^{N}\right)$ denote the Fourier series coefficients for $\tilde{C}_{1}\left\{u^{N}\left(t ; q^{N}\right)\right\}$ where $u^{N}$ is the solution to the initial value problem (4.3) corresponding to $q^{N}$ and let $U(k ; \bar{q})$ denote the Fourier series coefficients for $\tilde{C}_{1}\{u(t ; \bar{q})\}$ where $u$ is the solution to the initial value problem (2.1) corresponding to $\bar{q}$. If $\tilde{C}_{1}\left\{u^{N}\left(t ; q^{N}\right)\right\} \rightarrow \tilde{C}_{1}\{u(t ; \bar{q})\}$ in $V$ norm, and pointwise evaluation is continuous in the $V$ norm, then

$$
\sum_{\ell=1}^{\bar{N}_{M}}\left(\epsilon_{1}\left|f_{k_{\ell}^{u^{N}}}\left(q^{N}\right)-f_{k_{\ell}^{u}}(\bar{q})\right|^{2}+\epsilon_{2} \sum_{j=-n_{\ell}}^{N_{\ell}}|| U^{N}\left(k_{\ell}^{u^{N}}+j ; q^{N}\right)|-| U\left(k_{\ell}^{u}+j ; \bar{q}\right)||^{2}\right) \rightarrow 0
$$

as $N \rightarrow \infty$.

Now we are ready to state the main theorem for parameter estimation in the frequency domain formulation.

Theorem 4 Assume that the parameter space $Q$ is a compact subset of Euclidean space. Then each of the approximating estimation problems for (4.2) has a solution $\bar{q}^{N}$. Moreover, the sequence $\left\{\bar{q}^{N}\right\} \subset Q$ admits a convergent subsequence $\left\{\bar{q}^{N_{j}}\right\}$ with $\bar{q}^{N_{j}} \rightarrow \bar{q} \in Q$ as $j \rightarrow \infty$. If for each $q \in Q, U(k ; q)$ is defined as in Theorem 3 , then $\bar{q}$ is a solution to the original optimization problem for (3.5).

For the proof of both these theorems, see [7].

Continuous dependence of parameter estimates on observations (an analogue of the "method stability" of [1], [5]) can be established for frequency domain estimation problems using the ideas above with the arguments given in [1], [5] and [7]. 
Next we consider conditions under which $\tilde{C}_{1}\left\{u^{N}\left(t ; q^{N}\right)\right\}$ would converge to $\tilde{C}_{1}\{u(t ; q)\}$ in $V$-norm. For the following theorems, some assumptions on the finite dimensional spaces $H^{N}$ are required. We assume

$$
H^{N} \subset V \subset H
$$

(A6) For each $z \in V$, there exists $\hat{z} \in H^{N}$ such that $\left|z-\hat{z}^{N}\right|_{V} \rightarrow 0$ as $N \rightarrow \infty$.

Theorem 5 Suppose both $\sigma_{1}(q)$ and $\sigma_{2}(q)$ in (2.2) satisfy the conditions (A1)-(A3) and that conditions (A5)-(A6) hold. Let $q^{N}$ be arbitrary such that $q^{N} \rightarrow q$ in $Q$. Then

$$
\mathcal{T}^{N}\left(t ; q^{N}\right) P_{\mathcal{H}}^{N} \xi \rightarrow \mathcal{T}(t ; q) \xi, \quad \xi \in \mathcal{H}, t>0
$$

and

$$
\mathcal{A}^{N}\left(q^{N}\right) \mathcal{T}^{N}\left(t ; q^{N}\right) P_{\mathcal{H}}^{N} \xi \rightarrow \mathcal{A}(q) \mathcal{T}(t ; q) \xi, \quad \xi \in \mathcal{H}, t>0
$$

in $\mathcal{V}$ norm, with the convergence being uniform in $t$ on compact subintervals. Here $\mathcal{T}^{N}(t ; q)$ and $\mathcal{T}(t ; q)$ are the analytic semigroups generated by $\mathcal{A}^{N}(q)$ and $\mathcal{A}(q)$, respectively.

For a proof of this theorem, see [3], [6].

Corollary 1 Let $\mathcal{A}^{N}(q)$ and $\mathcal{A}(q)$ be the infinitesimal generators of the analytic semigroups $\mathcal{T}^{N}(t ; q)$ and $\mathcal{T}(t ; q)$, respectively, and $f \in C^{1}([0, T] ; V)$. Then for $\tilde{C}_{1}$ in one of the forms: identity, $d / d t$ or $d^{2} / d t^{2}$ we have

$$
\tilde{C}_{1}\left\{u^{N}\left(t ; q^{N}\right)\right\} \rightarrow \tilde{C}_{1}\{u(t ; q)\}
$$

in $V$-norm, where $u^{N}\left(t ; q^{N}\right)$ and $u(t ; q)$ are the first coordinate of $(4.4)$ and (2.5) respectively.

This corollary follows from Theorem 5 . For the detailed proof when $\tilde{C}_{1}=d^{2} / d t^{2}$ see $[6]$.

Before concluding this section, some comments on the assumption (A4) are appropriate. All our parameter estimation investigations have shown (and simple analysis of $2^{\text {nd }}$ order damped scalar systems suggest) that the frequencies of the vibration of a beam are primarily determined by parameters representing stiffness, mass density of the beam and mass of the tip body, whereas the magnitude of each excited mode is determined by the damping parameters (as well as the excitation force, of course). Stiffness and mass can be measured and calculated quite accurately through the experiments and these values can be used in the process of parameter 
identification (ID). That is, those measured quantities can be used as initial values to begin optimization. Using (4.2) as a cost function, we have carried out our parameter ID using the following three steps to ensure that (A4) was satisfied. First, we fix damping parameters with values from our knowledge of previous experience with experimental configurations similar to the one being studied. We use the measured stiffness and mass as initial values and optimize on those parameters. Then we fix the stiffness and mass parameters at the optimal values resulting from the first step and optimize on the damping parameters. Finally, we proceed to carry out an optimization on all parameters using the optimal values of the parameters from the previous steps as an initial guess. Our numerical efforts with such a procedure here (and in previously reported findings) have proved most satisfactory.

The basic mathematical model that we have considered in connection with the efforts discussed in this paper is the Timoshenko equations for a cantilevered beam with tip body. We shall describe the model in some detail in the next section.

\section{$5 \quad$ Example}

As an example, we apply the techniques outlined above to the flexural vibrations of elastic beams represented by models that include the effects of rotary inertia and shear deformation. We consider a cantilevered beam with tip body, internal or material damping, and an applied transverse force. The partial differential equation together with boundary conditions based on the Timoshenko theory in terms of the bending moment $M(t, x)$ and the shear force $S(t, x)$ are given by (see [8], [9], [13], [14] and $[15])$

$$
\begin{aligned}
& \rho \frac{\partial^{2} u}{\partial t^{2}}(t, x)-\frac{\partial S}{\partial x}(t, x)+\gamma \frac{\partial u}{\partial t}(t, x)=\tilde{f}(t, x), \\
& \rho r^{2} \frac{\partial^{2} \alpha}{\partial t^{2}}(t, x)-\frac{\partial M}{\partial x}(t, x)-S(t, x)=0, \quad 0<x<\ell, t>0 \\
& m c \frac{\partial^{2} u}{\partial t^{2}}(t, \ell)+\left(J_{o}+m c^{2}\right) \frac{\partial^{2} \alpha}{\partial t^{2}}(t, \ell)+M(t, \ell)=0, \quad t>0 \\
& m \frac{\partial^{2} u}{\partial t^{2}}(t, \ell)+m c \frac{\partial^{2} \alpha}{\partial t^{2}}(t, \ell)+S(t, \ell)=0, \quad t>0, \\
& u(t, 0)=\alpha(t, 0)=0, \quad t>0 .
\end{aligned}
$$

Here $\rho$ is the linear mass density, $u(t, x)$ is the transverse displacement, $\alpha(t, x)$ is the

rotation of the beam cross section, $\tilde{f}(t, x)$ is the external applied transverse forces, $r^{2}=I / A$ where $I$ is the moment of inertia of the cross sectional area $A$ and $\ell$ is 
the length of the beam. In (5.1), viscous (air) damping has been taken into account with $\gamma$ as damping coefficient. We have assumed that the tip body has mass $m$ and moment of inertia $J_{o}$ about its center of mass which is assumed to be located at a distance $c$ from the tip of the beam along the beam's axis or centerline. The bending moment and shear force with Kelvin-Voigt damping are given by

$$
\begin{aligned}
& M(t, x)=E I \frac{\partial \alpha}{\partial x}(t, x)+c_{D} I \frac{\partial^{2} \alpha}{\partial t \partial x}(t, x), \\
& S(t, x)=\kappa A G \beta(t, x)+\kappa A c_{s} \frac{\partial \beta}{\partial t}(t, x),
\end{aligned}
$$

where $G$ is the shear modulus, $\kappa$ is a correction factor, the shear distortion $\beta(t, x)$ is defined by $\partial u(t, x) / \partial x-\alpha(t, x), c_{D} I$ is the bending damping coefficient, and $c_{s}$ represents resistance related to shear strain rate. The possible parameters of the system to be considered are

$q=\left(\rho, A, G, E I, m, c, J_{o}, \gamma, c_{D} I, c_{s}\right) \in Q \subset \mathbb{R}^{10}$. In view of the physical meaning of each parameter, the admissible parameter set will be taken to be a compact subset of $\mathbb{R}^{10}$ with $c, \gamma \geq 0$ and each of $\rho, A, G, E I, m, J_{o}, c_{D} I, c_{s}$ bounded below by some positive constants.

The Hilbert spaces $H$ and $V$ are defined by $H=\mathbb{R}^{2} \times H^{0}(0, \ell) \times H^{0}(0, \ell)$ with inner product for $\Phi=\left(\nu_{1}, \nu_{2}, \phi_{1}, \phi_{2}\right), \Psi=\left(\xi_{1}, \xi_{2}, \psi_{1}, \psi_{2}\right) \in H$

$$
<\Phi, \Psi>_{\boldsymbol{H}}=<\Lambda\left(\nu_{1}, \nu_{2}\right),\left(\xi_{1}, \xi_{2}\right)>_{\mathbb{R}^{2}}+<\rho \phi_{1}, \psi_{1}>+<\rho r^{2} \phi_{2}, \psi_{2}>
$$

where $\Lambda$ is given by

$$
\Lambda=\left(\begin{array}{cc}
m & m c \\
m c & m c^{2}+J_{o}
\end{array}\right),
$$

and $V=\left\{\left(\nu_{1}, \nu_{2}, \phi_{1}, \phi_{2}\right) \in H \mid \phi_{i} \in H_{L}^{1}(0, \ell), \nu_{i}=\phi_{i}(\ell), i=1,2\right\}$ with inner product for $\Phi, \Psi \in V$

$$
<\Phi, \Psi>_{V}=<\left(D \phi_{1}-\phi_{2}\right),\left(D \psi_{1}-\psi_{2}\right)>+<D \phi_{2}, D \psi_{2}>
$$

Here we use the notation $D=\partial / \partial x$ and $H_{L}^{1}(0, \ell)=\left\{\phi \in H^{1}(0, \ell) \mid \phi(0)=0\right\}$. A normalized variational form of (5.1) has the same form as (2.2)

$$
\begin{aligned}
& <\ddot{z}(t), \Phi>_{V^{*}, V}+\sigma_{1}(q)(z(t), \Phi)+\sigma_{2}(q)(\dot{z}(t), \Phi)=<f(t), \Phi>_{H} \quad \forall \Phi \in V \\
& z(0)=0 \\
& \dot{z}(0)=0
\end{aligned}
$$


with

$$
\begin{aligned}
z(t) & =(u(t, 1), \alpha(t, 1), u(t, \cdot), \alpha(t, \cdot)) \\
\Phi & =\left(\phi_{1}(1), \phi_{2}(1), \phi_{1}, \phi_{2}\right) \\
f(t) & =\left(0,0, \rho^{-1} \tilde{f}(t, \cdot), 0\right),
\end{aligned}
$$

and

$$
\begin{aligned}
\sigma_{1}(q)(z(t), \Phi)= & <\kappa A G(D u-\alpha),\left(D \phi_{1}-\phi_{2}\right)>+<E I D \alpha, D \phi_{2}> \\
\sigma_{2}(q)(\dot{z}(t), \Phi)= & <\kappa A c_{s}(D \dot{u}-\dot{\alpha}),\left(D \phi_{1}-\phi_{2}\right)> \\
& +<c_{D} I D \dot{\alpha}, D \phi_{2}>+<\gamma \dot{u}, \phi_{1}>
\end{aligned}
$$

The first order abstract form of (5.1) for $w(t)=(z(t), \dot{z}(t))^{T}$ is

$$
\dot{w}(t)=\mathcal{A}(q) w(t)+F(t)
$$

where

$$
\mathcal{A}(q)=\left(\begin{array}{cc}
0 & I \\
-A(q) & -B(q)
\end{array}\right)
$$

with

$$
\begin{aligned}
A(q) z(t)=( & \Lambda^{-1}(-\kappa A G(\alpha(1)-D u(1)), E I D \alpha(1)), \\
& \rho^{-1} D(\kappa A G(\alpha-D u)), \\
& \left.\left(\rho r^{2}\right)^{-1}(D(E I D \alpha)-\kappa A G(\alpha-D u))\right), \\
B(q) \dot{z}(t)=( & \Lambda^{-1}\left(-\kappa A c_{s}(\dot{\alpha}(1)-D \dot{u}(1)), c_{D} I D \dot{\alpha}(1)\right), \\
& \rho^{-1} D\left(\kappa A c_{s}(\dot{\alpha}-D \dot{u})\right)+\rho^{-1} \gamma \dot{u}, \\
& \left.\left(\rho r^{2}\right)^{-1}\left(D\left(c_{D} I D \dot{\alpha}\right)-\kappa A c_{s}(\dot{\alpha}-D u)\right)\right) .
\end{aligned}
$$

The domain of $\mathcal{A}(q)$ is defined by

$$
\begin{gathered}
\operatorname{dom}(\mathcal{A}(q))=\left\{\chi=(\Phi, \Psi) \in V \times H \mid \Psi \in V, E I D \phi_{2}+c_{D} I D \psi_{2} \in H^{1}(0,1),\right. \\
\left.\kappa A G\left(\phi_{2}-D \phi_{1}\right)+\kappa A c_{s}\left(\psi_{2}-D \psi_{1}\right) \in H^{1}(0,1)\right\} .
\end{gathered}
$$

With the chosen parameter space, both $\sigma_{1}(q)$ and $\sigma_{2}(q)$ satisfy (A1)-(A3) hence $\mathcal{A}(q)$ generates an analytic semigroup.

A Galerkin method can be applied in developing an approximation scheme (see [3], [15] for details) with cubic splines chosen to generate a set of basis elements 